\title{
Erratum: Big Black Brain Phenomenon: Understanding Clinicoradiological Dissociation in Non-Accidental Traumatic Brain Injury in Children
}

Nitya Beriwal ${ }^{1}$, Albert L. Misko ${ }^{2}$, Ann-Christine Duhaime ${ }^{3}$

1. Medicine, Lady Hardinge Medical College, New Delhi, IND 2. Department of Neurology, Massachusetts General Hospital and Harvard Medical School, Boston, USA 3. Department of Neurosurgery,

Massachusetts General Hospital and Harvard Medical School, Boston, USA

Corresponding author: Nitya Beriwal, beriwalnitya@icloud.com

Corresponding author: Nitya Beriwal

\begin{abstract}
1. Medicine, Lady Hardinge Medical College, New Delhi, IND 2. Department of Neurology, Massachusetts General Hospital and Harvard Medical School, Boston, USA 3. Department of Neurosurgery, Massachusetts General Hospital and Harvard Medical School, Boston, USA

How to cite this erratum

Beriwal N, Misko A L, Duhaime A (May 14, 2020) Correction: Big Black Brain Phenomenon: Understanding Clinicoradiological Dissociation in Non-Accidental Traumatic Brain Injury in Children. Cureus 12(5): c31. doi:10.7759/cureus.c31
\end{abstract}

\section{Correction Notice}

The abstract was originally included twice and this duplication was, unfortunately, not caught by our copy editors. We have removed the duplicated text. 